\title{
Testicular Mixed Choriocarcinoma and Yolk Sac Tumor
}

National Cancer Institute

\section{Source}

National Cancer Institute. Testicular Mixed Choriocarcinoma and Yolk Sac Tumor. NCI

Thesaurus. Code C67548.

A malignant germ cell tumor arising from the testis. It is characterized by a mixture of choriocarcinoma and yolk sac tumor morphologic elements. Patients may present with painless or painful testicular swelling. 